\title{
Challenging mitral paravalvular leak and recurrent infective endocarditis
}

\author{
Katarzyna Elżbieta Gil', Christine Jellis², Amar Krishnaswamy³ \\ The Ohio State University Division of Cardiovascular Medicine, Columbus, Ohio, USA \\ 2Section of Cardiovascular Imaging, Heart and Vascular Institute, Cleveland Clinic, Cleveland, Ohio, USA \\ ${ }^{3}$ Section of Invasive and Interventional Cardiology, Heart and Vascular Institute, Cleveland Clinic, Cleveland, Ohio, USA
}

\author{
Correspondence to: \\ Katarzyna Elżbieta Gil \\ $\mathrm{MD}, \mathrm{PhD}$, \\ The Ohio State University \\ Division of Cardiovascular \\ Medicine, \\ 452 W 10 th Ave, Columbus, \\ $43210 \mathrm{OH}$, United States \\ phone: +16149075600 \\ e-mail: \\ katarzyna77.gil@gmail.com \\ Copyright by the \\ Author(s), 2021 \\ Kardiol Pol. 2021; \\ 79 (7-8): 885-886; \\ DOI: $10.33963 /$ KP.a2021.0016 \\ Received: \\ April 19, 2021 \\ Revision accepted \\ May 19, 2021 \\ Published online: \\ May 21, 2021
}

A 49-year-old man with hypertension, diabetes, and chronic renal failure on dialysis was treated with cardiac surgery involving coronary bypass and Medtronic Mosaic bioprosthetic mitral valve (MV) replacement for MRSA infective endocarditis (IE) at another institution. He presented a month later with decompensated heart failure due to severe paravalvular mitral regurgitation with hemolytic anemia.

Transthoracic echocardiography (TTE) demonstrated a focal echodensity on the ventricular side of the MV prosthesis (Figure 1A, arrow). Blood cultures were negative and there were no systemic features of sepsis. Transesophageal echocardiography (TEE) clarified that the echodensity was actually a distorted prosthetic valve strut, which was seemingly bent around outside of the annulus and protruding into the left atrium (Figure 1B-C, arrows). This was creating two paravalvular leaks (PVL) (posteromedially at 5 o'clock and posterolaterally at 8 o'clock in the surgeon's view), which in combination resulted in severe mitral regurgitation (Figure $1 D$, arrows). There were no clinical or echocardiographic findings to suggest IE.

The hospital course was complicated by the hemorrhagic shock which required embolization of the right inferior segmental renal artery and midzone interlobar artery. Due to his comorbidities, the patient was felt to be a highrisk redo surgical candidate. He, therefore, underwent percutaneous PVL closure (PVLC) under conscious sedation. 3DTEE was essential for guidance of this procedure, which required the deployment of six closure devices around the posterior aspect of the prosthetic valve annulus (Figure 1E, arrow) to successfully reduce the regurgitation to mild. Hemolytic anemia resolved following the procedure.
Three months later the patient presented with MRSA septic shock likely due to an infected dialysis catheter. TTE and TEE confirmed good PVLC results and revealed a decrease in left ventricular ejection fraction from $60 \%$ to $30 \%$ without findings suggestive of IE. Cardiac computed tomography (CT) revealed a fluid collection in the anterior chest wall. Radiolabelled white blood cell single-photon emission CT(SPECT)/CT found an abnormal uptake within the anterior chest wall confirming the presence of a sternal abscess and nonspecific uptake in the MV (Figure 1F, arrow). Sternal wound debridement was performed. The decision was made to defer MV replacement due to high surgical risk, negative blood cultures, and lack of definite IE diagnosis and IE complications on imaging studies. Follow-up TTE revealed an interval increase in LVEF to $55 \%$.

The incidence of PVL after surgical valve replacement is $2 \%-10 \%$ in the aortic position and $7 \%-17 \%$ in the mitral position $[1,2] .1 \%-5 \%$ of PVL cause serious clinical complications including hemolytic anemia, heart failure, and IE $[1,2] .3 D$ TEE is the gold standard for PVL evaluation [1]. PVLC is a safe and less invasive alternative to surgical reintervention for patients who are deemed unsuitable for surgery [2]. PVLC is associated with a high success rate and lower morbidity and mortality [1,2]. Active IE is a contraindication to PVLC, although the diagnosis of prosthetic valve IE is often challenging [3]. Radiolabelled white blood cell SPECT/CT increases the specificity of the modified Duke criteria in prosthetic valve $\mathrm{IE}$, especially in the case of recent cardiac surgery and foreign body implantation, although the uptake in this patient was nonspecific, and there were no other findings to suggest IE [4]. 


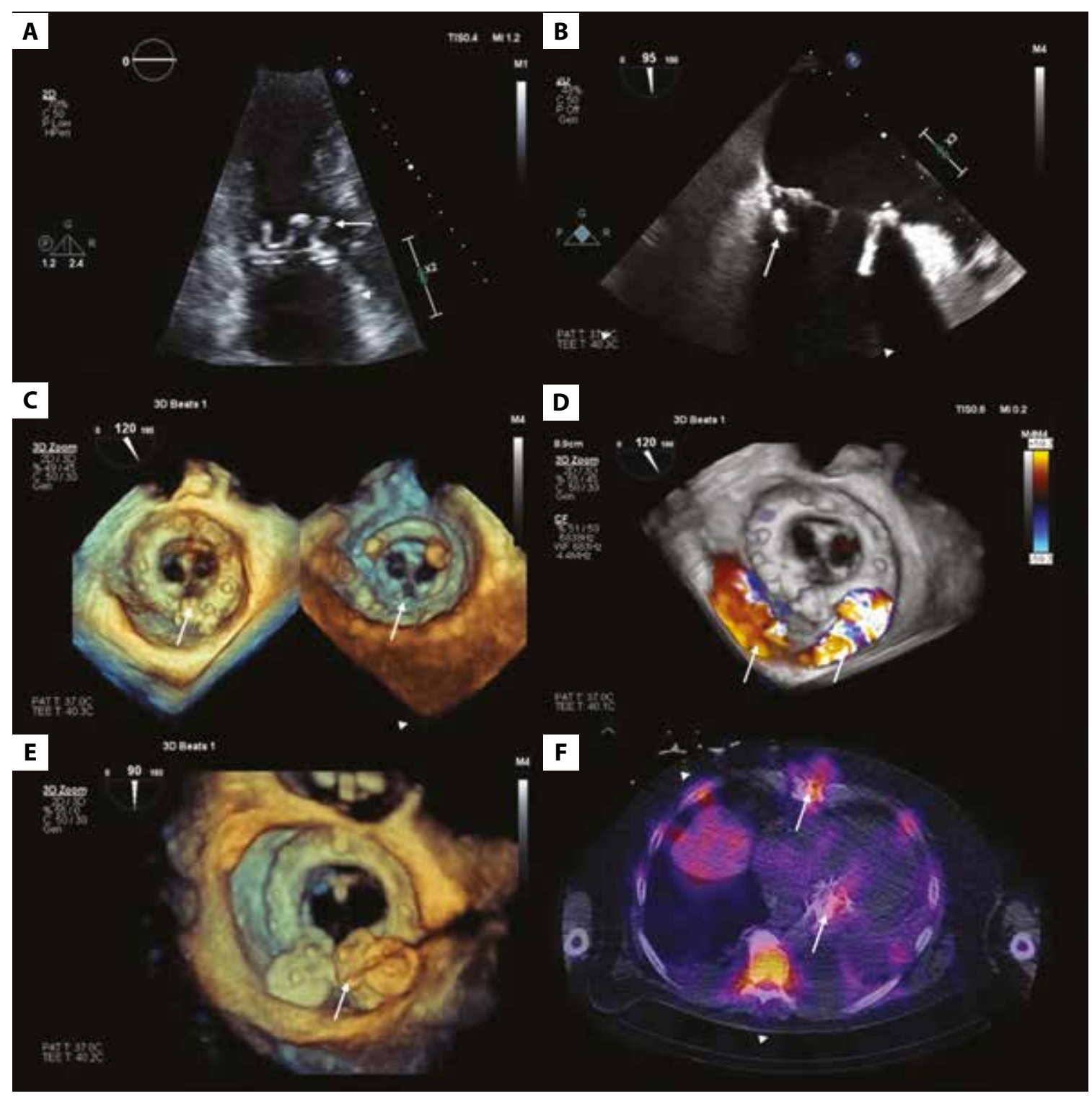

Figure 1. Percutaneous paravalvular leak closure. A. Transthoracic echocardiographic three-chamber view revealing a focal echodensity on the left-ventricular side of the mitral valve prosthesis concerning vegetation or thrombus (arrow). B. and C. 2D and 3D transesophageal echocardiographic views showing a bent prosthetic valve strut (arrows). D. Resultant severe paravalvular mitral regurgitation (arrows). E. Final result of percutaneous paravalvular leak closure with six closure devices under 3D transesophageal echocardiography guidance (arrows). F. Radiolabelled white blood cell SPECT/CT scan revealing abnormal uptake within the anterior chest wall and nonspecific focal uptake along with the prosthetic mitral valve (arrows)

Abbreviations: CT, computed tomography; SPECT, single-photon emission CT

\section{Article information}

Conflict of interest: None declared.

Open access: This article is available in open access under Creative Common Attribution-Non-Commercial-No Derivatives 4.0 International (CC BY-NC-ND 4.0) license, allowing to download articles and share them with others as long as they credit the authors and the publisher, but without permission to change them in any way or use them commercially. For commercial use, please contact the journal office at kardiologiapolska@ptkardio.pl.

How to cite: Gil KE, Jellis C, Krishnaswamy A. Challenging mitral paravalvular leak and recurrent infective endocarditis. Kardiol Pol. 2021; 79(7-8): 885-886, doi: 10.33963/KP.a2021.0016.

\section{REFERENCES}

1. Cruz-Gonzalez I, Rama-Merchan JC, Rodríguez-Collado J, et al. Transcatheter closure of paravalvular leaks: state of the art. Neth Heart J. 2017; 25(2): 116-124, doi: 10.1007/s12471-016-0918-3, indexed in Pubmed: 27878547.

2. Bouhout I, Ghoneim A. Rethinking mitral paravalvular leak closure: surgical approach or plugging the holes? JACC Cardiovasc Interv. 2017; 10(19): 1957-1958, doi: 10.1016/j.jcin.2017.08.032, indexed in Pubmed: 28982559.

3. Eleid MF, Cabalka AK, Malouf JF, et al. Techniques and outcomes for the treatment of paravalvular leak. Circ Cardiovasc Interv. 2015; 8(8): e001945, doi: 10.1161/CIRCINTERVENTIONS.115.001945, indexed in Pubmed: 26206850.

4. Cahill TJ, Baddour LM, Habib G, et al. Challenges in infective endocarditis.J Am Coll Cardiol. 2017; 69(3): 325-344, doi: 10.1016/j.jacc.2016.10.066, indexed in Pubmed: 28104075. 